\title{
Hyper-resistivity Theory in a Cylindrical Plasma
}

\author{
H. L. Berk, T. K. Fowler, L. L. LoDestro, and L. D. \\ Pearlstein
}

February 27, 2001

U.S. Department of Energy

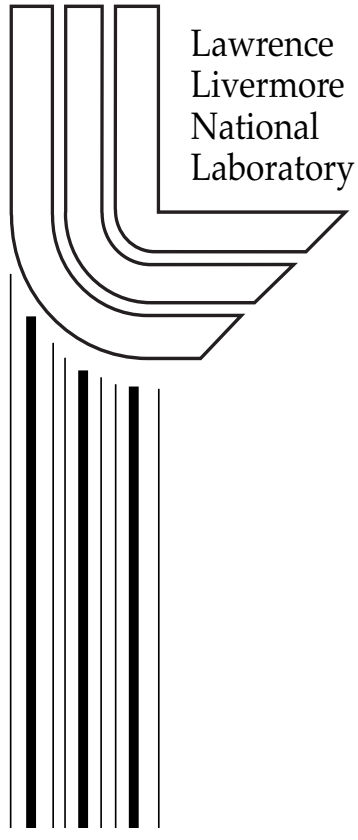




\section{DISCLAIMER}

This document was prepared as an account of work sponsored by an agency of the United States Government. Neither the United States Government nor the University of California nor any of their employees, makes any warranty, express or implied, or assumes any legal liability or responsibility for the accuracy, completeness, or usefulness of any information, apparatus, product, or process disclosed, or represents that its use would not infringe privately owned rights. Reference herein to any specific commercial product, process, or service by trade name, trademark, manufacturer, or otherwise, does not necessarily constitute or imply its endorsement, recommendation, or favoring by the United States Government or the University of California. The views and opinions of authors expressed herein do not necessarily state or reflect those of the United States Government or the University of California, and shall not be used for advertising or product endorsement purposes.

This work was performed under the auspices of the U. S. Department of Energy by the University of California, Lawrence Livermore National Laboratory under Contract No. W-7405-Eng-48.

This report has been reproduced directly from the best available copy.

Available electronically at http://www.doc.gov/bridge

Available for a processing fee to U.S. Department of Energy

And its contractors in paper from

U.S. Department of Energy

Office of Scientific and Technical Information

P.O. Box 62

Oak Ridge, TN 37831-0062

Telephone: (865) 576-8401

Facsimile: (865) 576-5728

E-mail: reports@adonis.osti.gov

Available for the sale to the public from

U.S. Department of Commerce

National Technical Information Service

5285 Port Royal Road

Springfield, VA 22161

Telephone: (800) 553-6847

Facsimile: (703) 605-6900

E-mail: orders@ntis.fedworld.gov

Online ordering: http://www.ntis.gov/ordering.htm

OR

Lawrence Livermore National Laboratory

Technical Information Department's Digital Library

http:/ / www.llnl.gov/tid/Library.html 


\title{
Hyper-resistivity Theory in a Cylindrical Plasma
}

\author{
H.L. Berk \\ Institute for Fusion Studies, The University of Texas, Austin, TX 78712 \\ T.K. Fowler, L.L. LoDestro, and L.D. Pearlstein* \\ Lawrence Livermore National Laboratory, P.O. Box 808 (L-630), Livermore, CA 94551-9990
}

\begin{abstract}
A model is presented for determining the hyper-resistivity coefficient that arises due to the presence of magnetic structures that appear in plasma configurations such as the reversed field pinch and spheromak. Emphasis is placed on modeling cases where magnetic islands pass from non-overlap to overlap regimes. Earlier works have shown that a diffusion-based model can give realistic transport scalings when magnetic islands are isolated, and this formalism is extended to apply to the hyper-resistivity problem. In this case electrons may either be in long or short mean-free-path regimes and intuitively-based arguments are presented of how to extend previous theories to incorporate this feature in the presence of magnetic structures that pass from laminar to
\end{abstract}

*Work performed under the auspices of the U.S. Department of Energy by University of California Lawrence Livermore National Laboratory under contract No. W-7405-Eng-48. 
moderately chaotic regimes.

Typeset using REVTEX 


\section{INTRODUCTION}

This paper develops a hyper-resistive transport equation that can be applied to the magnetic field evolution of reversed field pinches (RFP's) and spheromaks. The mechanism for hyper-resistivity is often attributed to the presence of chaos in the magnetic field structure of a plasma, and the basic framework for this description was first described in [1], who used chaotic (or braided) magnetic field concepts developed earlier by Zaslovski, et al. [2], Stix [3], and Rechester and Rosenbluth [4]. These approaches assume that the magnetic field form a chaotic sea, a situation that may not always apply in actual experiment, where when there is good confinement, there can be magnetic island structures that are separated by regions of good magnetic flux surfaces. Alternatively, a physical system can exhibit two different phases, a time interval when there are good magnetic surfaces and a time interval when there is a chaotic sea. The goal of this paper is to develop a set of transport equations that can bridge both limits. A similar problem was discussed in relation to kinetic instabilities [5], when there is only modest enhanced transport, when KAM surfaces separate kinetic islands (see for example, Ref. [6]) that have emerged from particle resonances, while transport is global and strongly enhanced when the islands are large enough to cause island overlap that break KAM surfaces. Initially, the formalism developed in Ref. [5] is applied to the magnetic field problem, but this formalism needs to be extended in order to incorporate physically important regimes and to make contact with the theory of Ref. [4] which applies to the case when the field lines are chaotic and finite electron mean-free-path along field lines are important.

In this paper we will primarily address the development of chaotic fields due to the nonlinear growth of tearing mode instabilities [7] whose evolution has been described by Rutherford [8] for a low-beta plasma. In this case plasma inertial effects are minimized and electron dynamics along a field line dominate the nonlinear response. We will further simplify the formal study by limiting its development to a cylindrical plasma, although we will speculate on the applicability of our results to more realistic geometry. Thus we neglect 
other interesting MHD-dynamics that might govern hyper-resistivity [e.g. Refs. [9-12]. A more complete transport theory, such as is discussed in Ref. [13], and built upon in Ref. [14] still needs to be incorporated to describe a general turbulent transport framework in a toroidal plasma.

The issue of the effect of a finite number of magnetic islands has been discussed by several authors [15-17]. The approach closest to ours is presented in Ref. [17], but we obtain somewhat different results. In our work we match the results of the long mean-free-path limit to the Rechester and Rosenbluth diffusion coefficient given in Ref. [4]. Such matching is not achieved in Ref. [17] (see further discussion of this point in Sec. IV).

Our formalism begins with a fairly systematic approach that develops a quasi-linear theory with a finite number of islands using the method developed in Ref. [5]. That method linked conventional quasi-linear theory, when there is strong resonance overlap, to a quasilinear theory of a single island that is governed by a diffusion equation. The treatment of the latter is based on rigorous arguments when modest collision effects are present but becomes more intuitive in the collisionless limit. Nonetheless in Refs. [2] and [5] it was shown that the diffusion captured many (though not all) of results rigorously derived from single island equations $[2,18,19]$. As our physical modeling is not rigorous, it is not surprising when some expected conservation laws do not emerge from our formalism. As an example, we nearly reproduce a magnetic helicity conservation structure $[11,20,21]$ which has been used by Boozer [22] to obtain a differential conservation structure. Our theory differs from the Boozer form only in non-dominant terms, and we modify in an ad-hoc manner the final equation to cast the final result in the Boozer conservation form. Other, constraints violated are energy, momentum and perhaps intrinsic particle flux ambipolarity [23-25]. (The key to demonstrating these conservation properties in weak perturbation theory is to incorporate non-resonant terms into the quasi-linear equation and to couple their response to the mode dynamics; a procedure that is readily incorporated with so-called perturbative modes [26] but which has not been totally solved for non-perturbative modes such as nonlinear tearing 
modes.)

\section{OVERVIEW OF PAPER}

The structure of the paper is as follows. In Sec. III we begin by presenting a theory that is direct generalization of the method used in Ref. [5], and we obtain the quasi-linear response of electrons with a long mean-free-path to very low frequency transverse magnetic perturbations (that characterize low beta tearing modes). We study the response of electrons to an isolated magnetic island. The electrons can traverse a thin island by cross-field transport. The theory presented in this work uses a Krook model with an effective collision frequency that can be synchronized to the results of a Fokker-Planck equation as described in Ref. [19]. However, once electrons can circulate around the magnetic island (assuming long mean-freepath along the field lines) faster than they can cross it by diffusive processes, the description of the response through a diffusion equation losses its rigor. Nonetheless, in Refs. [2,5] it was shown that a description where there is a large diffusion coefficient over a limited region of phase space produces physically reasonable scalings and we assume a similar applicability applies to our problem. When the isolated island response is extended to many modes using the standard random phase assumption, we obtain a global quasi-linear equation.

In Sec. IV several issues are developed in a physically intuitive manner. One is to compare the diffusion equation obtained when there are many modes, with the magnetic diffusion coefficient found for a stochastic magnetic field as described in Ref. [2]. To the extent electrons move along field lines without collisions or only cross-field collision processes are accounted for, we find that the results of the work presented here replicate the results of Refs. [2] and [5]. When collisions that impede motion along a field line are taken into account we may need to generalize the physical description. This arises when electrons can circulate around the island through many random steps faster than they can move across the magnetic island through cross field processes. We describe in a heuristic manner the physics processes that then need to be accounted for to reproduce the short mean path form 
of Rechester-Rosenbluth diffusion [4]. Another study of how to bridge the gap between long and short mean-free-paths of particles in stochastic fields is given in Ref. [16].

In Ref. [4] the magnetic correlation length, (the size of the mean-free-path compared to the magnetic correlation length is what determines the long or short mean-free-path limit in Rechester-Rosenbluth theory) is not precisely defined. This problem has been studied in Ref. [27] and in general there is not a universal expression for the correlation length. We point out that if the magnetic field patterns are only moderately chaotic, so that magnetic island structure still persists as part of the chaotic fields, the magnetic correlation length will be comparable to the circumferential length of an island and this length is used for in our theory. Then, when there is island overlap, we obtain a diffusion coefficient similar to that of Rechester and Rosenbluth but with a correlation length that is dependent on the magnitude of the perturbed magnetic fields.

Finally all these results are incorporated into a quasi-linear equation that is applied to a wide range of parameters. The first moment of this equation is taken to form an ohm's law that contains a hyper-resistivity term and thereby gives a current-diffusion equation. A brief discussion is given of how the current-diffusion equation can in principle be solved simultaneously with the $\Delta^{\prime}$ parameter used in both linear [7] and nonlinear [8] tearing mode theory. Then we can in principle determine the spectrum and saturation level of tearing modes, which in turn modifies the current diffusion equation. The numerical investigation of these equations will complement direct computational studies of nonlinear resistive MHD codes such as NIMROD [28] and other MHD studies [29] to understand dynamo-like behavior in RFP's and spheromaks. 


\section{ELECTRON RESPONSE TO MAGNETIC ISLANDS (FORMAL PRESENTATION)}

\section{A. Perturbed magnetic field}

We begin with the response to a single island. First, if there are no field perturbations, the magnetic vector potentials and magnetic fields are given by

$$
\boldsymbol{A}=\Phi \boldsymbol{\nabla} \theta-\psi \boldsymbol{\nabla} \phi
$$

where $\Phi$ and $\psi$ are respectively the toroidal (for arbitrarily large aspect ratio) and poloidal flux. The magnetic field is

$$
\begin{aligned}
\boldsymbol{\nabla} \times \boldsymbol{A} & =\boldsymbol{\nabla} \Phi \times \boldsymbol{\nabla} \theta-\boldsymbol{\nabla} \psi \times \boldsymbol{\nabla} \phi=B_{\phi} \widehat{\boldsymbol{\phi}}+B_{\theta} \widehat{\boldsymbol{\theta}} \\
\Phi & =\int_{0}^{r} d r r B_{\phi}, \quad \psi=\int_{0}^{r} d r R B_{\theta}
\end{aligned}
$$

with $r$ the minor radius, $R$ the major radius $(R \gg r)$ and $2 \pi R=L$ is the periodicity length.

We introduce a single resonant helical perturbation with an amplitude proportional to

$\exp (i m \xi)$ with $\xi=\theta-\frac{n}{m} \phi$. We look in a region where $\boldsymbol{b} \cdot \boldsymbol{\nabla} \exp [i m \xi] \approx 0$, so that we are considering a resonant perturbation. If the perturbation is small enough, the radial scale length of the perturbation can be ignored, the perturbed field is represented by a radial field $\delta \boldsymbol{B} \perp \boldsymbol{b}_{0}$, with $\boldsymbol{b}_{0}=\boldsymbol{B}_{0} / B_{0}$ and $\boldsymbol{B}_{0}$ the unperturbed magnetic field. With perturbations, the unit vector $\boldsymbol{b}$ is of the form, $\boldsymbol{b}=\boldsymbol{b}_{0}+\delta \boldsymbol{B} / B$.

To treat this case it is convenient to modify the vector potential to the form

$$
\begin{aligned}
\boldsymbol{A} & =\Phi \boldsymbol{\nabla} \theta-\psi \boldsymbol{\nabla} \phi \\
& =\Phi \nabla \xi-\widetilde{\psi} \boldsymbol{\nabla} \phi
\end{aligned}
$$

with

$$
\widetilde{\psi}=\left(\psi-\frac{n}{m} \Phi\right) \doteq-\frac{\delta r^{2}}{2}\left(\frac{B_{\phi}}{q} s\right)_{n, m}+\widetilde{\psi}_{0}
$$


where $\widetilde{\psi}_{0}$ is a constant that can be ignored as it does not affect the magnetic field, $\delta r=$ $\left(r-r_{n, m}\right)$ and $n, m$ refer to resonance surface where $\boldsymbol{b}_{0} \cdot \boldsymbol{\nabla} \exp (i m \xi)=0$. Note that at $r=r_{n, m}, q=q_{n, m}, \frac{n B_{\phi}\left(r_{n, m}\right)}{R}-\frac{m B_{\phi}\left(r_{n, m}\right)}{r_{n, m}}=0, q_{n, m}=\left.\frac{r B_{\phi}}{R B_{\theta}}\right|_{n, m}$, and $s=\frac{r}{q} d q /\left.d r\right|_{n, m}$.

Now a perturbation of the form $-\frac{2 \delta B_{r} R r_{n, m} \cos m \xi}{m} \boldsymbol{\nabla} \phi$ is added to the vector potential. Hence we find,

$$
\boldsymbol{A}=\Phi \nabla \xi-\tilde{\chi} \boldsymbol{\nabla} \phi
$$

with

$$
\tilde{\chi}=\left[-\frac{\delta r^{2}}{2}+\frac{2 \delta B_{r} R r_{n, m}}{m} \cos m \xi\right]\left(\frac{s B_{\phi}}{q}\right)_{n, m} .
$$

The magnetic field is

$$
\boldsymbol{B}=\nabla \times \boldsymbol{A}=\boldsymbol{\nabla} \Phi \times \boldsymbol{\nabla} \xi-\widehat{\boldsymbol{\theta}} \frac{\delta r}{R}\left(\frac{s B_{\phi}}{q}\right)_{n, m}+\widehat{\boldsymbol{r}} 2 \delta B_{r} \sin m \xi .
$$

\section{B. Kinetic response of electrons to magnetic perturbations}

Now let us consider the drift kinetic equation for electrons. We will use coordinates where $\mu$ (magnetic moment) and $v_{\|}$and $R_{g}$ (guiding center) are the variables. We neglect electron flows except the dominant one along the field line. We then have,

$$
\frac{\partial f}{\partial t}+v_{\|} \boldsymbol{b} \cdot \nabla f-C(f)=-\frac{e}{m_{e}} \boldsymbol{E} \cdot \boldsymbol{b} \frac{\partial f}{\partial v_{\|}}
$$

where $C(f)$ is the collisional operator and $e$ the electron charge. To lowest order we neglect the fine-scale structure of the perturbed field $\delta B_{r}$, so that $\boldsymbol{b}=\boldsymbol{b}_{0}$. The lowest order solution is

$$
f=f_{0}(\Phi, \boldsymbol{v})
$$

where $f_{0}(\Phi, \boldsymbol{v})$ is close to a Maxwellian that is needed to balance the collisional term, but with a small departure to allow for an equilibrium current generated by either a mean inductive field and hyper-resistive effects that are to be calculated in this paper. 
To next order, we account for the fine-scale perturbed magnetic field, and Eq. (6) becomes

$$
\frac{\partial f_{1}}{\partial t}+v_{\|} \boldsymbol{b}_{0} \cdot \nabla f_{1}-C\left(f_{1}\right)=-\frac{e}{m_{e}} E_{\|} \frac{\partial f_{1}}{\partial v_{\|}}-v_{\|} \frac{\delta \boldsymbol{B}}{B} \cdot \boldsymbol{\nabla} f_{0}
$$

or in $\xi$ and $\phi$ coordinates

$$
\begin{gathered}
\frac{\partial f_{1}}{\partial t}+v_{\|} \frac{B_{\phi}}{B R} \frac{\partial f_{1}}{\partial \phi}+v_{\|}\left(-\frac{n}{m} \frac{B_{\phi}}{B R}+\frac{B_{\theta}}{r B}\right) \frac{\partial f_{1}}{\partial \xi}-C\left(f_{1}\right)+\frac{e}{m_{e}} E_{\| 0} \frac{\partial f_{1}}{\partial v_{\|}} \\
=-\frac{e}{m_{e}} E_{\| 1} \frac{\partial f_{0}}{\partial v_{\|}}+\left(i v_{\|} \frac{\delta B_{r}}{B} \widehat{\boldsymbol{r}} \cdot \nabla f_{0} e^{i m \xi}+\text { c.c. }\right) .
\end{gathered}
$$

Note that

$$
\left(\frac{B_{\theta}}{r}-\frac{n}{m} \frac{B_{\phi}}{R}\right) \approx-\frac{\delta r}{R}\left(\frac{B_{\phi} s}{q r}\right)_{m, n}
$$

To solve this equation we assume that the perturbed magnetic fields can be treated as quasistationary, so that $\frac{\partial f_{1}}{\partial t}$, and the inductive field $E_{\| 1}$, associated with the time dependence of the perturbed fields, can be ignored. Further, note that we may have $C\left(f_{1}\right) \gtrsim \nu f_{1}$ (where $\nu$ is the electron scattering rate) because $C\left(f_{1}\right)$ proportional to a second derivative in either guiding center space [discussed below after Eq. (13)] or velocity space. Thus we will sometimes neglect $\nu$, although we will see there are important regimes when it needs to be considered. On the other hand the $E_{\| 0}$ term is balanced by the rate the electromagnetic skin effect changes, which is even much smaller than $\nu$. Thus, $E_{\| 0}$ can be neglected in Eq. (9).

In the appendix we consider Eq. (8) in its nonlinear limit, when $f_{0}$ and $f_{1}$ are replaced by the total $f$ and collisional and electric field terms are ignored (but when there can be an overall current in the global equilibrium). The result of this analysis is that electrons move around an island at a typical rate (we will not be concerned with precise numerical factors),

$$
\omega_{t r} \approx \frac{v_{t h e}}{B}\left(\frac{2 s m B_{\phi} \delta B_{r}}{q R r}\right)_{m, n}^{1 / 2}
$$

and the magnetic island width $\Delta_{\text {Isl }}$ is,

$$
\Delta_{\text {Isl }}=\left(\frac{2 \delta B_{r} q R r}{s B_{\phi} m}\right)_{m, n}^{1 / 2} .
$$


We now solve Eq. (9) by first assuming that electron diffusion takes a particle across the island width $\Delta_{\text {Isl }}$ at a rate that is faster than $\omega_{t r}$. If we treat the collisional term as a Krook operator of the form, $\nu_{\text {eff }} f_{1}$, we obtain the solution,

$$
\begin{aligned}
f_{1} & =\frac{-v_{\|} \delta B_{r} e^{i m \xi} \widehat{\boldsymbol{r}} \cdot \nabla f}{i \nu_{\mathrm{eff}}+\delta r\left(m v_{\|} s B_{\phi} / q B r R\right)_{m, n}}+\text { c.c. } \\
& =\frac{i \delta B_{r} e^{i m \xi} v_{\|} \widehat{\boldsymbol{r}} \cdot \nabla f \nu_{\mathrm{eff}}}{\nu_{\mathrm{eff}}^{2}+\delta r^{2}\left(m v_{\|} s B_{\phi} / q B r R\right)_{m, n}^{2}}+\text { c.c. }+ \text { (nonresonant contribution) } .
\end{aligned}
$$

To estimate the magnitude of the Krook operator, we use that in guiding center coordinates the form of the collision term is $C(f) \simeq \nu \rho^{2} \nabla^{2} f \sim \nu \rho^{2} f / \delta r^{2}$, where $\rho$ is the electron Larmor radius, and $\nu$ the $90^{\circ}$ scattering rate of an electron (this procedure is discussed more fully in Ref. [19]). Near resonance this term is balanced by the term

$$
\frac{\delta r}{B R}\left(\frac{B_{\phi} s}{q r}\right)_{m, n} v_{\|} \frac{\partial f}{\partial \xi} \approx \frac{\delta r v_{\|}}{R} \frac{m B_{\phi} s}{q r B} f
$$

giving

$$
\delta r \sim\left(\nu \rho^{2} \frac{q r R B}{m B_{\phi} s v_{\|}}\right)^{1 / 3}
$$

Thus,

$$
\nu_{\text {eff }} f \sim \frac{\nu \rho^{2} f}{\delta r^{2}} \approx \nu^{1 / 3}\left(\frac{\rho s m B_{\phi} v_{t h e}}{q R r B}\right)^{2 / 3} f
$$

where the electron thermal velocity is used for $v_{\|}$. We should note that this method of estimation is valid if $\delta r$ in Eq. (14) is greater than the magnetic island width $\Delta_{\text {Isl }}$ given in Eq. (12). Otherwise, the exact solution discussed in the appendix is relevant. Then its effect can be captured in an approximate way in the manner described in Ref. [19]. Specifically we add to $\nu_{\text {eff }}$, determined in Eq. (15), the term $\omega_{t r}$ from Eq. (11). Hence, for $\nu_{\text {eff }}$ we use,

$$
\nu_{\mathrm{eff}}=\nu^{1 / 3}\left(\frac{\rho s m B_{\phi} v_{t h e}}{q R r B}\right)_{m, n}^{2 / 3}+v_{t h e}\left(\frac{2 s m B_{\phi} \delta B_{r}}{q r R B^{2}}\right)_{m, n}^{1 / 2}=\nu_{\mathrm{eff} 0}+\omega_{t r} .
$$

We note that the resonance term for $f_{1}$ given in Eq. (13), has Lorentzian localization. It may be that the localization should even be sharper, especially when it is determined by $\omega_{t r}$ in Eq. (16). 
The "quasi-linear" response is obtained by iterating the Vlasov equation to next order, to find the spatially homogeneous response. We obtain, keeping collisions and the mean inductive field,

$$
v_{\|} \boldsymbol{b}_{0} \cdot \nabla f-C(f)+\frac{e}{m} \boldsymbol{E} \cdot \boldsymbol{b}_{0} \frac{\partial f}{\partial v_{\|}}-\frac{i v_{\|}}{B} \boldsymbol{\nabla} \cdot\left(\delta \boldsymbol{B}_{r} f_{1} e^{i m \xi}+\text { c.c. }\right) f_{1}=0 .
$$

We have kept the $v_{\|} \boldsymbol{b}_{0} \cdot \nabla f$ term for formal reasons that are important when we have several

resonances present. If we substitute for $f_{1}$ in Eq. (14), and integrate by $\int \frac{d s}{v_{\|}}$over a surface, we obtain

$$
-C(f)+\frac{e}{m} \boldsymbol{E} \cdot \boldsymbol{b}_{0} \frac{\partial f}{\partial v_{\|}}=\frac{v_{\|}^{2}}{B} \nabla \cdot \frac{\widehat{\boldsymbol{r}} 2\left|\delta B_{r}\right|_{m, n}^{2} \nu_{\mathrm{eff}} \widehat{\boldsymbol{r}}}{\left[\nu_{\mathrm{eff}}^{2}+\delta r_{m, n}^{2}\left(\frac{m v_{\|} s B_{\phi}}{q B r R}\right)_{m, n}^{2}\right] B_{m, n}} \cdot \nabla f .
$$

Subsequently we suppress the subscripts of $\delta r$ and we assume the two terms on the left-hand side of Eq. (18) are insensitive to the averaging procedure. If there were several resonances, the above procedure is readily generalized assuming a random phase approximation, to give,

$$
-C(f)+\frac{e}{m} \boldsymbol{E} \cdot \boldsymbol{b}_{0} \frac{\partial f}{\partial v_{\|}}=\frac{v_{\|}^{2}}{B^{2}} \nabla \cdot \sum_{(n, m)} \frac{\widehat{\boldsymbol{r}} 2\left|\delta B_{r}\right|_{m, n}^{2} \nu_{\mathrm{eff}} \widehat{\boldsymbol{r}}}{\left[\nu_{\mathrm{eff}}^{2}+\left(\delta r \frac{s m v_{\|}}{r R q} \frac{B_{\phi}}{q B}\right)_{m, n}^{2}\right]} \cdot \nabla f .
$$

(Note, we have removed $1 / B$ from inside the integral to conform with the ultimate magnetic helicity conservation form we wish to obtain.)

\section{ELECTRON RESPONSE TO MAGNETIC ISLANDS}

\section{A. Heuristic considerations for the effect of finite mean-free path along field lines}

At this stage the procedure has been relatively formal. We see that we have obtained a diffusive response to the magnetic perturbations which is quite smooth if $\left|\nu_{\text {eff }}\right|>$ $\Delta r_{m, n}\left(\frac{m v_{\|}}{r R q} \frac{s B_{\phi}}{B}\right)$ where $\Delta r_{m, n}$ is the radial separation between resonant surfaces. On the other hand in the opposite limit, the radial diffusive response is very localized to the resonant surface. The result would be that radial diffusion is inhibited where the diffusion coefficient is small. 
Before continuing with our main line of inquiry, let us touch base with the previously considered problem, essentially discussed in Ref. [2]. There a spectrum of many modes is considered, where the distance along the field lines plays the role of time. This is exactly equivalent to our case in the limit of extremely low collisionality. For our problem, where $d \ell=v_{\|} d t$, we obtain a diffusion coefficient $D=\left|v_{\|}\right| D_{m}$, where $D_{m}$ is the magnetic diffusion coefficient of Ref. [2].

With many modes, and negligible collisional effects one can readily argue that the term $v_{\|} \boldsymbol{b}_{0} \cdot \nabla f$, in Eq. (17) is more accurately represented by $v_{\|} \boldsymbol{b} \cdot \nabla f$ with $\boldsymbol{b}$ evaluated with the omission of just one of the resonant terms at the particular radius that entangled magnetic fields have taken a field line to. With enough perturbative components of the field in the spectrum, this value for $\boldsymbol{b}$ is arbitrarily close to the direction of the true magnetic field. When we treat the limit where $\nu_{\text {eff }} \rightarrow 0$, and assume the radial gradients are weak, we can annihilate the $v_{\|} \boldsymbol{b} \cdot \nabla f$ term by averaging over the trajectory $\int \frac{d s}{v_{\|}}$, and we obtain, with the definition $\langle G\rangle=\int_{0}^{L} \frac{d s G}{v_{\|}} / L$,

$$
-\langle C(f)\rangle+\frac{e}{m_{e}}\left\langle\boldsymbol{E} \cdot \boldsymbol{b} \frac{\partial f}{\partial v_{\|}}\right\rangle=\lim _{\nu_{\mathrm{eff}} \rightarrow 0} \frac{1}{B^{2}} \boldsymbol{\nabla} \cdot \widehat{\boldsymbol{r}}\left\langle\sum_{m, n} \frac{2\left|\delta B_{r}\right|^{2} \nu_{\mathrm{eff}} v_{\|}^{2}}{\left[\nu_{\mathrm{eff}}^{2}+\left(\delta r \frac{s m v_{\|}}{r R q} \frac{B_{\phi}}{q B}\right)_{m, n}^{2}\right]}\right\rangle \widehat{\boldsymbol{r}} \cdot \boldsymbol{\nabla} f .
$$

There are several weak links to the above procedure, as was also noted in Ref. [2]. We have assumed the annihilation procedure commutes with the radial gradient. More explicitly we write the bracketed term as,

$$
\begin{gathered}
\left\langle\lim _{\nu_{\mathrm{eff}} \rightarrow 0} \sum_{m, n} v_{\|} \frac{2\left|\delta B_{r}\right|_{m, n}^{2} \nu_{\mathrm{eff}} /\left|v_{\|}\right|}{\nu_{\mathrm{eff}}^{2} /\left|v_{\|}^{2}\right|+\left(\delta r m \frac{s B_{\phi}}{q B r R}\right)_{m, n}^{2}}\right\rangle \\
=\lim _{L \rightarrow \infty} \frac{2\left|\delta B_{r}\right|_{m, n}^{2} \frac{\nu_{\mathrm{eff}}}{v_{\|} \mid}}{\left|v_{\|}\right| \int_{0}^{L} d s \sum_{m, n} \frac{\left.\nu_{\mathrm{eff}}\right)^{2}+\left[\delta r m\left(s B_{\phi} / q B R r\right)_{m, n}\right]^{2}}{\left|v_{\|}\right|}} \equiv\left|v_{\|}\right| D_{M} . \\
\longrightarrow \pi \int d n \sum_{m} 2\left|\delta B_{r}\right|_{m, n}^{2}\left|v_{\|}\right| \delta\left(\delta r m s B_{\phi} /\left.q B R r\right|_{m, n}\right)
\end{gathered}
$$


$\left(\left|v_{\|}\right|\right.$arises since $s$ is integrated in the direction of $\left.v_{\|}\right)$. Equation (21) is basically the magnetic diffusion coefficient in a stochastic field obtained in Ref. [2]. A test particle at position $r_{0}$ where the magnitude of the mean field is $B_{0}$ diffuses radially as $\left\langle\left(r-r_{0}\right)^{2} B_{0}^{2}\right\rangle=D_{M}\left|v_{\|}\right| t$. To obtain this form we need to integrate in $L$ a distance greater than a correlation length $L_{\mathrm{c}}$, where $L_{\mathrm{c}}$ is the length along a field line beyond which the radial displacement becomes stochastic. It should be noted that

$$
D_{M} \approx \frac{\left(\delta B_{r} L_{c}\right)^{2}}{L_{c}}
$$

The precise form of the correlation length in the strongly overlapping regime is difficult to calculate, although it should be somewhat greater than $R$ and depend on magnetic shear. One also uses a formal procedure where the sum on $n$ is taken as an integral, so that a delta function then emerges that is independent of $\nu_{\text {eff }}$.

There is another complication to making contact with the result in Eq. (19) with previously analyzed results when there is appreciable mode overlap but when collisions need to be accounted for. We have assumed in our averaging over the stochastic field, that collisions do not affect the particle. In Ref. [4] collisions are accounted for both in the long mean-free path (mfp) limit, $L_{c} \ll L_{\mathrm{mfp}}$ with $L_{\mathrm{mfp}}$ the mean-free path, and short mfp limit $L_{\mathrm{mfp}} \ll L_{c}$. In the long mean-free-path limit, but where $L_{\mathrm{mfp}} \ll L_{\mathrm{mag}}$ (where $L_{\mathrm{mag}}$ is the length of the stochastic field line in the entire machine), Ref. [4] points out that $\left|v_{\|}\right|$can be replaced by $v_{\text {the }}$, the electron thermal speed, in Eq. (21), but only after an interesting subtlety is accounted for. This subtlety is due to the realization that electrons are not strictly constrained to remain on one dimensional "strings" that would allow an electron to return exactly to its starting point after a collision turns the electron around. It is pointed out that a cross-field process must be accounted for to cause the electron to feel a random phase when it goes back the same distance that it went forward. In particular, in Ref. [4], it is argued that cross-field diffusion from a random cross-field step the size of a Larmor radius causes phase decorrelation with respect to the initial and return paths if an electron moves along the field line a distance much greater than the correlation length $L_{\mathrm{c}}$. Other processes such as 
magnetic and curvature drifts as well as flow shear due to an otherwise small electric field can also cause the needed phase randomization. In our formulation, cross-field diffusion is built in from the very beginning. Hence, the replacement of $\left|v_{\|}\right|$by $v_{\text {the }}$ that is used in Ref. [4] is readily justified when mode overlap of magnetic islands exists.

There is still an additional subtlety that is particularly important when modes do not overlap. Let us then discuss the middle term in Eq. (21) (with $\left|v_{\|}\right|$replaced by $v_{t h e}$ ). We still assume long mfp, when $L_{\mathrm{mfp}} \ll v_{t h e} / \omega_{t r}$, but now we only have a single mode, with mode numbers $m$ and $n$. We explicitly display $\nu_{\text {eff }}$ as $\nu_{\text {eff }}=\nu_{\text {eff } 0}+\omega_{t r}$,

$$
D_{M}=\frac{2\left|\delta B_{r}\right|_{m, n}^{2}\left(\frac{\nu_{\text {eff } 0}}{v_{t h e}}+\frac{\omega_{t r}}{v_{t h e}}\right)}{\left[\left(\nu_{\mathrm{eff} 0}+\omega_{t r}\right) / v_{t h e}\right]^{2}+\left(\delta r m s B_{\phi} / q B R r\right)_{m, n}^{2}} .
$$

We note that if $\nu_{\text {eff } 0} \gg \omega_{t r}$, the radial region of diffusion, though limited in size, is larger than an island width (the diffusion zone, $\Delta_{\text {dif }}$ is $\Delta_{\text {dif }} \approx \Delta_{\text {Isl }}\left(\frac{\nu_{\text {eff } 0}}{\omega_{t r}}\right)$. Such scaling for the zone of diffusion is also predicted in more rigorous theory, where the radial diffusion operator is accurately solved. The difference of our heuristic model is only in the particular Lorentzian shape shape that the Krook model produces, while a more quantitative calculation would give a sharper cut-off to the diffusion zone compared to a relatively broad Lorentzian shaping factor.

When $\nu_{\text {eff } 0}$ is less than $\omega_{t r}$, we have a magnetic "diffusion" coefficient that is only appreciable in a distance $\Delta_{\text {dif }} \approx \Delta_{\text {Isl }}$, with $D_{M}$ roughly given by $D_{M} \approx B_{0}^{2} \Delta_{\text {Isl }}^{2} \omega_{t r} / v_{\text {the }}$. This intrinsic diffusivity scales independent of any electron dynamics as from Eq. (11) $\omega_{t r} / v_{t h e}=\left(2 s B_{0} \delta B_{r} / B^{2} q R r\right)^{1 / 2}$. The single island diffusion coefficient scales in the same way as the result calculated in Ref. [2], where sophisticated, but still approximate analysis was used. The interpretation of this diffusion coefficient in this regime is that in the diffusion zone there is a random radial step $\Delta_{\text {Isl }}$ when the magnetic field line traverses a circumferential distance $v_{t h e} / \omega_{t r} \sim B\left(q R r / s m B_{\phi} \delta B_{r}\right)^{1 / 2}$ around the island. However, we can expect that the effective diffusion coefficient should have a form factor much narrower than a Lorentzian as randomness in the magnetic field cannot exist much beyond an island separatrix. 
Thus the rules we have used produce a reasonable scaling law for diffusion within a single island, although in a strict sense there is in fact no diffusion when a magnetic island is well defined (indeed one can well argue that we are dealing with an internal contradiction). The same problem arises in more general dynamical systems when the problem can be reduced to considering the dynamics associated with an island emerging from a single nonoverlapping resonance. Nonetheless, it was shown [5] that there are a class of problems, such as the prediction of saturation levels, where the treatment of the island dynamics with this intuitively derived diffusion coefficient gives results that correlate well when compared with the predictions of more accurate descriptions. Thus with appropriate caution, we will use the diffusion coefficient we have calculated for a single island in the hyper-resistivity problem. In particular, we have in mind a dynamical system, where tearing modes cause $\delta B_{r}$ to slowly grow, and where there is a gradient in the current profile around the resonant surface. Then for an island growing sufficiently slowly that induction effects can be ignored, the current density formed from two open-field lines that have reconnected to form a closedisland region, has a current density that is the average of the current density that is the average of the surrounding open field lines. This means that the current density in the island is flat, independent of the surface in the island, with a rather steep current gradient arising at the surfaces that are adjacent to the open field lines. Somewhat outside the islands, the current density is close to the value calculated when there are no islands. This type of dynamical behavior is produced by our diffusion model, where the diffusion zone is limited to the region just surrounding the separatrix. This is also just the type of problem that was studied in Ref. [5], where phase space island dynamics was studied rather successfully using just this diffusion model.

We now need to discuss the important short mean-free-path limit. This problem was treated in Ref. [4], where the conclusion was reached that the radial electron diffusion in a stochastic magnetic field is reduced by a factor $L_{\mathrm{mfp}} / L_{c}$, where $L_{\mathrm{c}}$ is the correlation distance along a field line needed to make the magnetic field stochastic. As a result, if neglecting colli- 
sions the magnetic diffusion coefficient is $D_{M}$, which is given in terms of modal perturbations by the middle term of Eq. (21), and which also must have the form $D_{M} \approx \Delta r^{2} B_{0}^{2} / L_{\mathrm{c}}$, where $\Delta r$ is the random radial step taken by a field line after it moves a decorrelation distance $L_{\mathrm{c}}$ along a field line. Note that the correlation distance is in general difficult to calculate, and it will depend on the degree of overlap of the perturbed magnetic modes; further when islands just overlap the magnetic correlation length will scale as the circumferential length of the island $v_{t h e} / \omega_{t r} \sim B\left(q R r / s m B_{\phi} \delta B_{r}\right)^{1 / 2}$. Rechester and Rosenbluth conclude that for the short mean-free-path regime the radial diffusion coefficient will scale as

$$
D_{r} \sim \frac{v_{\text {the }} D_{M} L_{\mathrm{mfp}}}{B^{2} L_{\mathrm{c}}} \longrightarrow \frac{v_{\text {the }} D_{M} L_{\mathrm{mfp}}}{B^{2}\left(L_{\mathrm{c}}+L_{\mathrm{mfp}}\right)}
$$

where the last term is an interpolation between long and short mean-free-path limits.

We propose to continue to use a somewhat modified form of Eq. (23) when magnetic field lines are not completely stochastic, so that island structures can be resolved even when there is some overlap of magnetic islands and also if island overlap is not present. In this case the magnetic correlation length for both cases is $v_{t h e} / \omega_{t r}$. However, if the cross-field particle diffusion is fast enough so that the electron crosses the island transversely faster than it moves around the structure by following a field line (either by direct flight in the long mean-free-path limit or by multiple scatterings in the short mean-free-path limit), the radial diffusion coefficient should not change the diffusion structure from what we have already derived. Hence we propose that the appropriate model for the electron diffusion coefficient should be

$$
D_{r} \sim v_{t h e} D_{M} \frac{\left(\omega_{t r}+\nu_{\mathrm{eff} 0}\right)}{B_{0}^{2}\left(\omega_{t r}+\nu_{\mathrm{eff} 0}+\nu_{\|}\right)}
$$

where $\nu_{\|}$is the electron collision rate (the subscript $\|$has been added to emphasize that it arises from collisional effects that effect motion along a field line), with $D_{M}$ given by Eq. (21) (with $v_{\text {the }}$ replacing $\left|v_{\|}\right|$). It should be kept in mind that this form is sensible in the overlap regime only if island structure is clearly present [otherwise the estimate for the magnetic correlation length is incorrect as it should ultimately be insensitive to the form of $\delta B(r)]$. 
Similarly, the dependence on the enhanced perpendicular diffusion coefficient $\nu_{\text {effo }}$ should weaken considerably as the island structure disappears with increased perturbations.

Finally, we note that if we have an isolated island, and $\nu_{\|}>\omega_{t r}>\nu_{\text {eff } 0}$, we obtain a diffusion coefficient that scales as $D_{r} \sim \Delta_{\text {Isl }}^{2} / T_{1} \sim \Delta_{\text {Isl }}^{2} \omega_{t r}^{2} / \nu_{\|} \sim \nu_{\|}\left(L_{\text {mfp }} \delta B_{r} / B\right)^{2}$ where $T_{1} \approx \nu_{\|} / \omega_{t r}^{2}$ is the time for a particle to diffuse around the island after multiple collisional steps along a field line. This diffusion coefficient is based on the same rationale as the use of a diffusion coefficient for a particle transiting an isolated island in the long mean-free-path limit. The coefficient used here differs from that proposed in Ref. [17], which would be justified if an electron decorrelates from the magnetic island structure after one collision time. Apparently the justification for the model used in Eq. (17) is that the banana orbit width is larger than the island width. In our problem we assume the opposite is the case.

\section{B. Equation for hyper-resistivity}

Mindful of the caveats we have already noted, we propose the following diffusion coefficient to be used in all the regimes we intend to study,

$$
D_{r}=\frac{v_{t h e}^{2}}{B^{2}} \sum_{m, n} \frac{2\left|\delta B_{r}\right|^{2}\left(\nu_{\mathrm{eff} 0}+\omega_{t r}\right)^{2}}{\left[\left(\nu_{\mathrm{eff} 0}+\omega_{t r}\right)^{2}+\left(\frac{\delta r m v_{t h e} s B_{\phi}}{q r R B}\right)_{m, n}^{2}\right]\left(\nu_{\mathrm{eff} 0}+\omega_{t r}+\nu_{\|}\right)} .
$$

Now we proceed with constructing a hyper-resistivity theory that is valid over a wide range of parameters. Upon averaging over a surface, the resulting model equation becomes

$$
-C(f)+\frac{e}{m_{e}} \boldsymbol{E} \cdot \boldsymbol{b} \frac{\partial f}{\partial v_{\|}}=\lim _{\nu_{\mathrm{eff}} \rightarrow 0} \frac{1}{B^{2}} \boldsymbol{\nabla} \cdot \sum_{m, n} \frac{\widehat{\boldsymbol{r}} 2\left|\delta B_{r}\right|^{2} \nu_{\mathrm{eff}}^{\prime 2} v_{t h e}^{2} \widehat{\boldsymbol{r}}}{\left[\nu_{\mathrm{eff}}^{\prime 2}+\left(\frac{\delta r m v_{t h e} s B_{\phi}}{q B r R}\right)_{m, n}^{2}\right]\left(\nu_{\mathrm{eff}}^{\prime}+\nu_{\|}\right)} \cdot \nabla f
$$

where,

$$
\nu_{\mathrm{eff}}^{\prime} \approx \nu_{\perp}^{1 / 3}\left(\frac{\rho s m B_{\phi} v_{t h e}}{q R r B}\right)^{2 / 3}+\omega_{t r}
$$

and we have differentiated between collision processes along the field line, denoted by $\nu_{\|}$, and collision processes perpendicular to the field line, denoted by $\nu_{\perp}$. If other anomalous cross-field processes are present (e.g. cross-field diffusion due to drift wave turbulence) it 
can be modelled by increasing the value of $\nu_{\perp}$ above its classical value to an appropriate level.

Thus the right-hand side of Eq. (18), which we denote as the quasi-linear operator $Q_{L}(f)$ is altered to

$$
Q_{L}(f)=\frac{v_{\text {the }}^{2}}{B^{2}} \nabla \cdot \sum_{m, n} \frac{\widehat{\boldsymbol{r}} 2\left|\delta B_{r}\right|^{2} \nu_{\mathrm{eff}}^{\prime 2} \widehat{\boldsymbol{r}}}{\left(\nu_{\mathrm{eff}}^{\prime}+\nu_{\|}\right)\left[\nu_{\mathrm{eff}}^{\prime 2}+\left(\frac{\delta r m v_{t h e} s B_{\phi}}{r R q B}\right)_{m, n}^{2}\right]} \cdot \nabla f
$$

We now assess how successful we have been in obtaining an expression that can give hyper-resistivity with a finite number of modes. It turns out we are close, but we have to make one additional ad-hoc modification in order to obtain a form that will lead to a magnetic helicity conservation. We alter Eq. (28) slightly to obtain a kinetic equation of the form

$$
-C(f)+\frac{e}{m_{e}} \boldsymbol{E} \cdot \boldsymbol{b} \frac{\partial f}{\partial v_{\|}}=\frac{n_{e}}{B^{2}} \nabla \cdot \sum_{m, n} \frac{2\left|\delta B_{r}\right|_{m, n}^{2} \nu_{\mathrm{eff}}^{\prime 2} v_{t h e}^{2} \widehat{\boldsymbol{r}} \widehat{\boldsymbol{r}}}{n_{e}\left[\nu_{\mathrm{eff}}^{\prime 2}+\left(\delta r \frac{m v_{t h e} s B_{\phi}}{q B r R}\right)_{m, n}^{2}\right]\left(\nu_{\mathrm{eff}}^{\prime}+\nu_{\|}\right)} \cdot \nabla f
$$

with $n_{e}$ the electron density (the inverse of the electron density is placed inside the divergence operator to ultimately form the expected magnetic helicity conservation structure in the Ohm's law with hyper-resistivity). Now, when we take the moment of Eq. (29) (we integrate Eq. (28) by $\left.2 \pi e \int \frac{d \mu}{m_{e}} d v_{\|} v_{\|} B^{2}\right)$ with $\mu=m_{e} v_{\perp}^{2} / 2 B$, we find

$$
B E_{\|}=\eta j_{\|} B-\nabla \cdot \lambda \cdot \nabla\left(j_{\|} / B\right)
$$

where hyper-resistivity coefficient $\lambda$ is given by

$$
\lambda=\sum_{m, n} \frac{2\left|\delta B_{r}\right|_{m, n}^{2} \nu_{\mathrm{eff}}^{\prime 2} v_{t h e}^{2} m_{e}}{n_{e} e^{2}\left(\nu_{\mathrm{eff}}^{\prime 2}+\left[\delta r \frac{m v_{t h e} s B_{\phi}}{q B r R}\right]_{m, n}^{2}\right)\left(\nu_{\mathrm{eff}}^{\prime}+\nu_{\|}\right)} .
$$

Now the hyper-resistivity coefficient has the required form needed to conserve global magnetic helicity if the diffusion coefficient vanishes on the boundary. As asserted, to obtain this form we needed to make ad-hoc assumptions of what radial derivatives should be acted upon with regard to the mean magnetic field and mean density. However, it is important that in the short mean-free-path limit the driving term of the hyper-resistivity is proportional 
to the second derivative of $j_{\|} / B$. This means the hyper-resistivity term will attempt to flatten $j_{\|} / B$ even without the artificial shaping we used to obtain the hyper-resistivity term in conservation form.

It remains an open question, whether we can find deeper subtleties to our modeling, so as to better justify the precise conservation form of the hyper-resistivity term. One source of difficulty may be that the convection of magnetic helicity includes the magnetic helicity associated with the turbulent field. It is possible that in obtaining an equation for the evolution of the mean fields, precise magnetic helicity conservation does not emerge because we have not separated out the non-resonant component associated with the perturbing magnetic fields.

At any event, Eq. (30) seems to be a good starting point for a model that describes hyper-resistivity when there is a finite number of tearing modes. The next stage of the investigation is to study the equation for the mode evolution. For example, if modes do not couple, one can use Rutherford's theory for island growth [8], together with the solution of the hyper-resistivity equation (30) to obtain the form of the current profile with time. If the system has a small positive $\Delta^{\prime}$, (which may be the case if the system is only stable to tearing modes when there is only a small deviation from a fully relaxed Taylor state [30]), the saturation levels of the islands will be small when one applies rules established in the literature (e.g. see Ref. [31] for calculating $\Delta^{\prime}$ of a finite amplitude mode). Small island saturation is required for the theory we have developed. Still, with a limited number of modes, strong hyper-resistivity effects can arise when the modes grow to a large enough level to cause mode overlap. Further nonlinear modeling can be obtained by building upon mode-mode coupling theories, such as developed by Fitzpatrick [32] where the phases of the various tearing modes can lock and cause rotation, etc. These investigations will be subjects of future work. 


\section{DISCUSSION}

Hyper-resistivity may have important consequences for magnetic field evolution in RFP's and spheromaks. We have developed a coupled mode dynamics-diffusion model for these systems when they operate at high temperatures where they are expected to have well-defined magnetic flux surfaces most of the time, but with stochastic magnetic field entanglement arising periodically due to the development of unstable tearing modes. The magnetic entanglement can either be localized in space, or spread out over most of the contained plasma region. In our model the entanglement is needed to induce a dynamo-like action to sustain in the the mean the reversed toroidal magnetic field structure in an RFP, and to allow the magnetic helicity to diffuse into the core of a spheromak, where larger values of $j_{\|} / B$ are sustained at the edge with external electrodes.

Several important questions are of concern in the model we have developed. the first is that in order have rapid relaxation to a Taylor state by active tearing modes, then during the phase where magnetic stochasticity is present it is necessary for the hyper-resistive term to be larger than the usual Ohmic term. For establishing scaling we take $r / a \sim s \sim q \sim$ $B_{\phi} / B_{\theta} \sim 1$. There are basically two types of hyper-resistive relaxation modes, (a) partial relaxation where the island chain is over a fraction of the discharge and (b) total relaxation where the island chain spans the entire discharge. Our theory assumes that the magnetic islands, of radial width $\Delta_{\text {isl }} \sim\left(a R \delta B_{r} / B\right)^{1 / 2}$, only moderately overlap so that their identities are not destroyed during magnetic entanglement. Then, when there is relaxation of the entire discharge due to $N$ island structures that are roughly evenly spread out across the plasma, a maximum saturation of the magnetic field perturbation $\delta B_{r} / B \sim(a / R) / N^{2}$ is inferred.

Further, the theory we developed divides into a short mean-free-path limit, where

$$
\varepsilon_{1} \equiv L_{\mathrm{mfp}}\left(\delta B_{r} / B a R\right)^{1 / 2}<1
$$

and the long mean-free-path limit where $\varepsilon_{1}>1$. We will see that in the short mean-freepath-limit our theory does not predict rapid current relaxation due to the hyper-resistive 
term compared with the ohmic diffusion rate. However, in the long mean-free-path limit, rapid current relaxation due to the hyper-resistive mechanism we have described is possible.

First consider the short mean-free-path condition, $\varepsilon_{1}<1$, and compare the ratio, $r_{1}$ of the hyper-resistive to ohmic term in Eqs. (30) and (31). If partial relaxation arises, hyper-resistive diffusion acts in a radial region $\Delta_{w}$ where there is overlap, $\Delta_{w} \sim N \Delta_{\text {isl }} \sim$ $N\left(a R \delta B_{r} / B\right)^{1 / 2}$. Then we find,

$$
r_{1} \sim\left(\delta B_{r} L_{\mathrm{mfp}} / B \Delta_{w}\right)^{2} \sim \frac{\delta B_{r} L_{\mathrm{mfp}}^{2}}{B N^{2} a R}
$$

and if we require $r_{1}>1$ while satisfying Eq. (32), we must satisfy,

$$
\frac{a R}{L_{\mathrm{mfp}}^{2}}>\frac{\delta B_{r}}{B}>\frac{N^{2} a R}{L_{\mathrm{mfp}}^{2}}
$$

which is an incompatible condition as $N>1$. Thus this regime does not describe more rapid current profile relaxation from the hyper-resistive effect than from Ohmic diffusion. This difficulty persists even when the hyper-resistive diffusion acts on the entire current profile. In that case the saturation condition, $\delta B_{r} / B \sim(a / R) / N^{2}$, inherent our theory, leads to the same incompatibility condition given by Eq. (34).

Now we consider the long mean-free-path-regime, $\varepsilon_{1}>1$. When there is partial current profile relaxation, so that $\delta B_{r} / B<(a / R) / N^{2}$, the ratio of the hyper-resistive to ohmic terms $r_{2}$, is,

$$
r_{2}=\left(\frac{\delta B_{r}}{B}\right)^{3 / 2}(R a)^{1 / 2} L_{\mathrm{mfp}} / \Delta_{w}^{2} \sim L_{\mathrm{mfp}}\left(\frac{\delta B_{r}}{B}\right)^{1 / 2} /\left[N^{2}(R a)^{1 / 2}\right] \equiv \frac{\varepsilon_{1}}{N^{2}} .
$$

We then have rapid partial relaxation of the current profile compared with its Ohmic evolution rate if,

$$
a /\left(R N^{2}\right)>\delta B_{r} / B>N^{4} R a / L_{\mathrm{mfp}}^{2}
$$

We estimate $L_{\mathrm{mfp}} \sim 10^{4} T_{\mathrm{kev}}^{2} / n_{14} \mathrm{~cm}$. [ $T_{\mathrm{kev}}$ is the electron temperature in kev units, and $n_{14}$ is the particle density in units of $10^{14} \mathrm{~cm}^{-3}$ ). Then rewriting Eq. (36), with the major radius $R$ expressed as $R_{m}$ in meters, we have, 


$$
\frac{a}{\left(R N^{2}\right)}>\frac{\delta B_{r}}{B}>10^{-4} \frac{a}{R} N^{4} n_{14} \frac{R_{m}^{2}}{T_{\mathrm{kev}}^{4}} .
$$

Thus for a meaningful theory for rapid relaxation of the current profile to emerge from our theory we need $T_{\mathrm{kev}}>0.1 n_{14}^{1 / 2} R_{m}^{1 / 2} N^{3 / 2}$. For example with these estimates we have for an RFP with $N=3$, and $a / R \sim 0.25$, and $n_{14} \sim 0.5$,

$$
.025>\frac{\delta B_{r}}{B}>.0005 \frac{R_{m}^{2} n_{14}^{2}}{T_{\mathrm{kev}}^{4}} .
$$

This indicates a regime can be reached in an RFP (e.g. MST has $R_{m}=1.5$ and $T_{\mathrm{kev}} \approx$ 0.5) where rapid relaxation of the current profile due to hyper-resistivity can apply over a fraction of the current profile. The condition is somewhat more stringent for spheromaks, where $a / R \sim 1$ and we require for $N=3$,

$$
0.1>\frac{\delta B_{r}}{B}>2 \times 10^{-3} \frac{n_{14}^{2} R_{m}^{2}}{T_{\mathrm{kev}}^{4}} .
$$

For example for $R_{m} \sim 0.3$ and $n_{14} \sim 1.0$, we see that we need $T_{\text {kev }} \gtrsim 0.2 \mathrm{kev}$ to have a rapid relaxation caused by 3 modes over a fraction of the plasma. Experiments in the SSPX spheromak have produced $T_{\mathrm{kev}} \sim .12$ for $n_{14} \sim 1$, which is somewhat short of the estimate for when rapid relaxation in the long mean-free-path regime is applicable.

Thus we have demonstrated that if $\varepsilon_{1}$ is sufficiently large, we can obtain dominance of the hyper-resistivity term during relaxation of the current profile, and our model may be appropriate for present day experiments in an RFP, but that improved confinement parameters need to be obtained in spheromaks to test our theoretical model.

A potential difficulty with the hyper-resistive mechanism for controlled fusion application is that it implies exceeding rapid thermal diffusion over the region where current relaxation arises. The thermal diffusivity as given by the modified Rechester-Rosenbluth formula, Eq. (29) is

$$
D_{r}=v_{\text {the }}(R a)^{1 / 2}\left(\frac{\delta B_{r}}{B}\right)^{3 / 2} .
$$

The thermal relaxation time $\tau_{\text {thm }}$ over a radial width $\Delta_{w} \sim N \Delta_{\text {isl }} \sim N\left(a R \delta B_{r} / B\right)^{1 / 2}$ of overlapping islands is given by

$$
\tau_{\text {thm }} \sim \frac{\Delta_{w}^{2}}{D r} \sim \frac{N^{2}\left(a R B / \delta B_{r}\right)^{1 / 2}}{v_{\text {the }}} .
$$


The current relaxation rate $\nu_{\text {cur }}$ evaluated when hyper-resistive relaxation dominates conventional Ohmic relaxation is given by,

$$
\nu_{\text {cur }} \sim\left(\frac{\delta_{e m}}{\Delta_{w}}\right)^{2} \frac{r_{2} v_{t h e}}{L_{\mathrm{mfp}}} \sim \frac{\delta_{e m}^{2} v_{\text {the }}\left(B / \delta B_{r}\right)^{1 / 2}}{N^{4}(R a)^{3 / 2}}
$$

where $\delta_{e m}=c / \omega_{p e}$ is the electromagnetic skin depth. Thus $\nu_{\mathrm{cur}} \tau_{\mathrm{thm}} \sim \frac{\delta_{e m}^{2} B}{\delta B_{r} a R N^{2}}<\frac{\delta_{e m}^{2} L_{\mathrm{mfp}}^{2}}{\left(N^{3} a R\right)^{2}}$ is an extremely small number $\left(\delta_{e m} \sim .0005 n_{14}^{-1 / 2} \mathrm{~m}\right)$. Hence the temperature will flatten extremely rapidly over the region where magnetic islands overlap.

If the island chain does not reach the plasma edge, there can still be good thermal insulation. Indeed if $\Delta_{w} \ll a$, global heat confinement is hardly affected by local current relaxation. However, if the island chain reaches the edge, heat in the flattened temperature region is rapidly lost, though the loss rate is mitigated at the edge by the decrease of heat conduction that arises with a shortened mean-free-path and by an electron confining potential that forms at solid walls that open field lines reach. Hence at the edge, where there is an added effect of ohmic heating of a relatively high resistive plasma, one should maintain an electron temperature close to $100 \mathrm{ev}$. The difficulty is the possibility of a thermal collapse which can rapidly lower the temperature throughout the plasma to one that is close to the edge value.

Our theory does not demonstrate a strong current relaxation effect at low temperature that is characteristic of spheromak experiments that exist today [33]. Progress has been made in numerically simulating spheromak start-up conditions using the Nimrod code [28] and the results do not appear to exhibit reconnection as long as the gun voltage is applied. Instead a state forms that appears to evolve from laminar flow [34] in which field lines remain reconnected to the gun even though some lines become extended in length by coiling up inside the flux conserver [35]. However, in the actual experiment there may still be reconnection processes that are difficult to resolve in computer simulations, or even the physics of these reconnection processes may not be described by the simulation. It appears difficult for the theory developed in this work to treat this regime as it suppressed the effect of plasma flows which is likely to be extremely important during the formation phase. However, if 
reconnection does eventually form closed magnetic surface regions, the plasma should heat further, and eventually the model developed here should be applicable.

In experiments such as MST [36], magnetic flux surfaces are known to be well defined, and data indicates that tearing modes form and lead to dynamo-like action with abrupt current profile collapses. In these experiments our formalism is likely to have relevance, although we will need to further develop the theory to incorporate observed internal mode locking (to produce so-called slinky modes) that form from the nonlinear interaction of tearing modes.

\section{Acknowledgments}

We wish to thank Dr. Carl Sovinec for sharing advanced copies of a publication of Nimrod calculations in preparation, and Dr. Bruce Cohen of discussions of his Nimrod results. This work was supported by the U.S. Dept. of Energy Contract No. DE-FG03-96ER-54346. 


\section{Appendix: Solution to the single-mode problem}

In the helical mode problem we solve Eq. (8) neglecting $C(f)$ and $E_{\|}$. We seek a steady solution when we use $\xi$ and $r$ as variables, and expand close to the rationale surface, and $\boldsymbol{B} \cdot \widehat{\boldsymbol{r}}=2 \delta B_{r} \sin m \xi$. We find

$$
-v_{\|} \frac{\delta r}{B}\left(\frac{s B_{\phi}}{q r R}\right)_{m, n} \frac{\partial f}{\partial \xi}+\frac{2 v_{\|} \delta B_{r} \sin \xi}{B} \frac{\partial f}{\partial r}=0 .
$$

The equation allows a steady solution $f=f(\Phi, \tilde{\chi})$ with

$$
\tilde{\chi}=-\frac{\delta r^{2}}{2}\left(\frac{B_{\phi} s}{q}\right)_{m, n}+\frac{2 \delta B_{r} R r_{m, n} \cos m \xi}{m} .
$$

The actual dependence of $f(\Phi, \tilde{\chi})$ depends on detailed dynamics. However, one can imagine a limit where the mode amplitude grows slowly compared to the particle trapping frequency (to be estimated shortly below). In this case, the distribution will mix

$f\left(\Phi_{m, n}+\Delta \Phi\right)$ and $f\left(\Phi_{m, n}-\Delta \Phi\right)$ together, where $\Delta \Phi \approx B_{\phi} r d \Delta r$ and $\Delta r$ is the width of the separatrix (a more precise evaluation is possible, but the calculation will only change a numerical factor). As a result $f(\Phi, \chi)$ in the island region is flat and equal to $f\left(\Phi_{m, n}\right)$, surrounded by a region where $f$ is close to the unperturbed value $f(\Phi)$ with only a weak dependence on $\tilde{\chi}$.

To obtain the width of the island region we solve the characteristic equations,

$$
\frac{d \xi}{d t}=\frac{-v_{\|}}{B}\left(\frac{s B_{\phi}}{q r B}\right)_{m, n} \delta r, \quad \frac{d \delta r}{d t}=\frac{2 v_{\|} \delta B_{r} \sin m \xi}{B} .
$$

Combining these two equations leads to the pendulum equation,

$$
\frac{d^{2} \xi}{d t^{2}}=-2 v_{\|}^{2}\left(\frac{s B_{\phi}}{B^{2} q r R}\right)_{m, n} \delta B_{r} \sin m \xi
$$

The first integral of this equation is,

$$
\frac{1}{2}\left(\frac{d \xi}{d t}\right)^{2}-2 v_{\|}^{2}\left(\frac{s B_{\phi}}{q r R}\right)_{m, n} \frac{\delta B_{r} \cos m \xi}{m}=C .
$$

Particles are trapped in an island for 


$$
\frac{-2 v_{\|}^{2}}{m}\left(\frac{s B_{\phi}}{B^{2} q r R}\right)_{m, n} \delta B_{r}<C<\frac{2 v_{\|}^{2}}{m}\left(\frac{s B_{\phi}}{B^{2} q r R}\right)_{m, n} \delta B_{r}
$$

with a characteristic transit period, $\omega_{t r}$, (the trapping frequency of deeply trapped particles)

$$
\omega_{t r} \sim v_{\|}\left(\frac{2 s B_{\phi} m \delta B_{r}}{B^{2} q r R}\right)_{m, n}^{1 / 2}
$$

and the radial width of the island, $\Delta_{\mathrm{Isl}} \approx \frac{2 \delta B_{r}}{B} \frac{v_{\|}}{\omega_{t r}}$, is,

$$
\Delta_{\mathrm{Isl}} \approx\left(\frac{2 \delta B_{r} q r R}{s B_{\phi} m}\right)_{m, n}^{1 / 2}
$$

This solution gives the correct scaling as long as diffusion does not cause particles to cross the island structure at a rate $\nu_{\text {eff }}>\omega_{t r}$. This aspect of the problem is discussed further in the text. 


\section{REFERENCES}

[1] P.K. Kaw, E.J. Valeo, and P.H. Rutherford, Phys. Rev. Lett. 43, 1398 (1979).

[2] M.N. Rosenbluth, R. Z. Sagdeev, J.B. Taylor, and G., M. Zaslovski, Nucl. Fusion 6, 297 (1966).

[3] T.H. Stix, Phys. Rev. Lett. 36, 521 (1976), and Nucl. Fusion 18, 353 (1978).

[4] A. B. Rechester and M.N. Rosenbluth, Phys. Rev. Lett. 40, 38 (1978).

[5] H.L. Berk, B.N. Breizman, J. Fitzpatrick, and H.V. Wong, Nucl. Fusion 35, 1661 (1995).

[6] A.J. Lichtenberg and M.A. Lieberman, Regular and Stochastic Motion (Springer-Verlag, New York, 1983), p. 356.

[7] H.P. Furth, J. Killeen, and M.N. Rosenbluth, Phys. Fluids 6, 459 (1963).

[8] P.H. Rutherford, Phys. Fluids 16, 1903 (1973).

[9] D. Biskamp, Plasma Phys. Control. Fusion 26, 311 (1984).

[10] H.R. Strauss, Phys. Fluids 28, 2786 (1985).

[11] H.R. Strauss, Phys. Fluids 29, 3668 (1986).

[12] E. Hameiri and A. Bhattacharjee, Phys. Fluids 30, 1743 (1987).

[13] S.M. Mahajan, R.D. Hazeltine and D.A. Hitchcock, Phys. Fluids 26, 700 (1983).

[14] R. Gatto, Models of Coupled Transport to Investigate Anomalous Transport in Tokamaks, Ph.D. Dissertation, Dept. of Nucl. Eng., Univ. of Calif., Berkeley, 1996.

[15] C.C. Hegna and J.D. Callen, Phys. Fluids B 4.

[16] G. Laval, Phys. Fluids B 3, 711 (1993).

[17] R.H. Weening and A.H. Boozer, Phys. Fluids B 4, 159 (1992).

[18] H.L. Berk and B. N. Breizman, Phys. Fluids B 2, 2235 (1990). 
[19] H.L. Berk, B.N. Breizman, and M.S. Pekker, Plasma Phys. Rep. 9, 778 (1997).

[20] A. Bhattacharjee and E. Hameiri, Phys. Rev. Lett. 57, 206 (1986).

[21] M.A. Berger, Geophys. Astrophys. Fluid Dyn. 30, 79 (1984).

[22] A. H. Boozer, J. Plasma Phys. 35, 133 (1986).

[23] R. Waltz, Phys. Fluids 25, 1269 (1982).

[24] H.L. Berk and K. Molvig, Phys. Fluids 26, 1385 (1983).

[25] H.E. Mynick, J. Plasma Phys. 39, 303 (1988).

[26] Krall-Trivilpiece Textbook.

[27] J.A. Krommes, C. Oberman, and R. G. Kleva, J. Plasma Phys. 30, 11 (1983).

[28] A. H. Glasser, C. R. Sovinec, R. A. Nebel, T. A. Gianakon, S.J. Plimpton, M. S. Chu, D. D. Schnack, and the NIMROD Team, Plasma Phys. Control. Fusion 41, A747 (1999).

[29] S.C. Jardin, Nucl. Fusion 22, 629 (1982).

[30] J.B. Taylor, Rev. Mod. Phys. 58(3), 742 (1986).

[31] D. Biskamp, Nonlinear Magnetohydrodynamics (Cambridge Univ. Press, 1993), p. 119.

[32] R. Fitzpatrick, Phys. Plasmas 6, 1168 (1999).

[33] R.D. Wood, D.H. Hill, E.B. Hooper, B. Buchenauer, H. McLean, and S. Woodruff, to be published in J. Nucl. Mat. (2001).

[34] D. Montgomery and L. Phillips, Physica D 37, 215 (1989).

[35] R.E. Cardenas, S.T. Jones, C.C. Kim, S.E. Parker, and C. Sovinec, Bull. Am. Phys. Soc. 45(7), 180 (2000), (HP1-79).

[36] A.F. Almagri, S. Assadi, S.C. Prager, J.S. Sarff, and D.W. Kerst, Phys. Fluids B 4, 4080 (1992). 